Cinémas

Revue d'études cinématographiques

Journal of Film Studies

\title{
Pragmatique historique du film : Nouvelle Vague et conception de l'auteur. La réception d'À bout de souffle aux Pays-Bas
}

\section{Jürgen E. Müller}

Volume 2, numéro 2-3, printemps 1992

Cinéma et Réception

URI : https://id.erudit.org/iderudit/1001078ar

DOI : https://doi.org/10.7202/1001078ar

Aller au sommaire du numéro

Éditeur(s)

Cinémas

ISSN

1181-6945 (imprimé)

1705-6500 (numérique)

Découvrir la revue

Citer cet article

Müller, J. E. (1992). Pragmatique historique du film : Nouvelle Vague et conception de l'auteur. La réception d'À bout de souffle aux Pays-Bas. Cinémas, 2(2-3), 56-87. https://doi.org/10.7202/1001078ar
Résumé de l'article

Partant de la théorie de la pragmatique historique du texte, l'auteur développe un modèle heuristique d'une pragmatique historique du film. Basé sur l'esthétique de la réception, sur la phénoménologie et sur la sociologie de la connaissance, ce modèle s'attarde aux processus de production et de réception, c'est-à-dire au réseau d'activités qui constituent l'existence des « textes filmiques ". Dans la deuxième partie de l'article, l'auteur s'applique à démontrer les possibilités de son approche en analysant le rôle du concept de l'" auteur " (comme élément historique du savoir) dans la première réception du film $A$ bout de souffle aux Pays-Bas. 


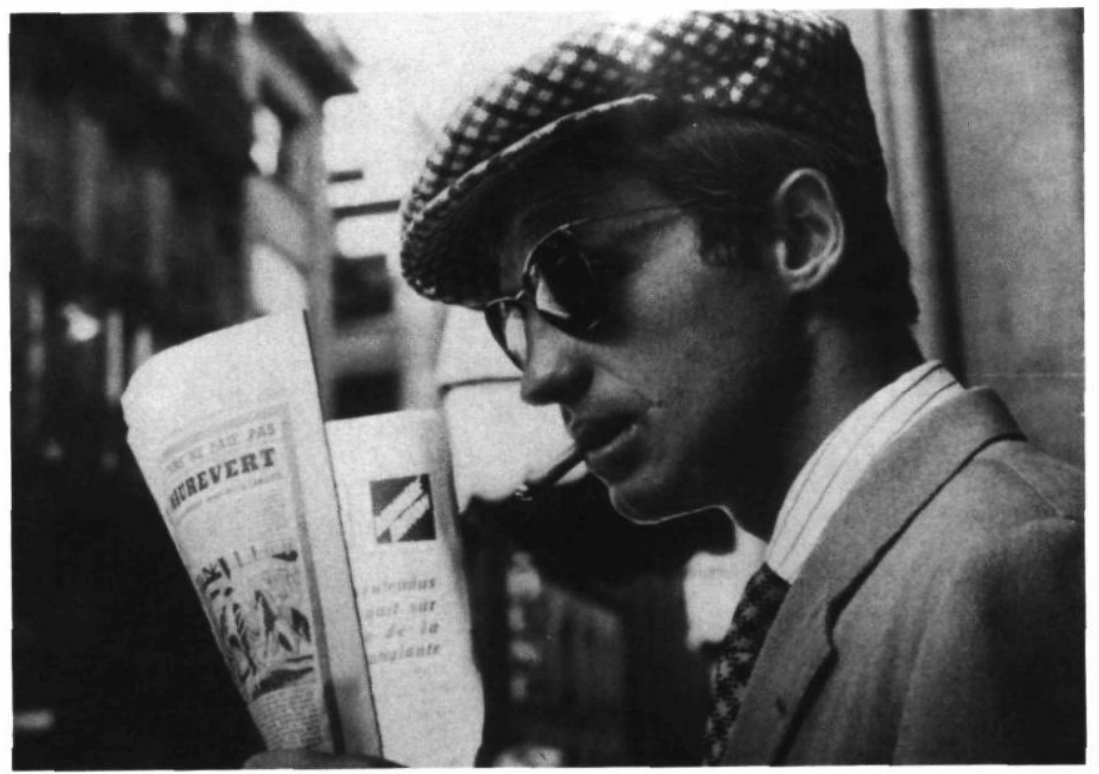

À bout de souffle de Jean-Luc Godard (1960) Coll.: Cinémathèque québécoise 


\title{
Pragmatique historique du film: Nouvelle Vague et conception de l'auteur. La réception $\mathrm{d}^{\prime} A$ bout de souffle aux Pays-Bas ${ }^{1}$
}

\section{Jürgen E. Müller}

\section{RÉSUMÉ}

Partant de la théorie de la pragmatique historique du texte, l'auteur développe un modèle heuristique d'une pragmatique historique du film. Basé sur l'esthétique de la réception, sur la phénoménologie et sur la sociologie de la connaissance, ce modèle s'attarde aux processus de production et de réception, c'est-à-dire au réseau d'activités qui constituent l'existence des «textes filmiques». Dans la deuxième partie de l'article, l'auteur s'applique à démontrer les possibilités de son approche en analysant le rôle du concept de l' «auteur» (comme élément historique du savoir) dans la première réception du film $\dot{A}$ bout de souffle aux Pays-Bas.

\begin{abstract}
Working from the theory of the historical pragmatics of text, the author develops a heuristic model for the historical pragmatics of film. Based on the aesthetics of reception, phenomenology, and the sociology of knowledge, this model focuses on the processes of production and reception, on the network of activities that constitute the existence of "filmic texts." In the second part of the article, the author demonstrates the possibilities of his model in an analysis of the role of the "author" concept (as a historical element of knowledge) in the initial reception of $\dot{A}$ bout de souffle in the Netherlands.
\end{abstract}




\section{Texte médiatique et action sociale}

L'affirmation selon laquelle il convient de situer la production et la réception de textes médiatiques dans des situations spécifiques et historiques de communication est aujourd'hui un lieu commun dans la recherche sur les textes et les films. Malgré toutes les différences quant à son fondement théorique qui s'étend de l'herméneutique à la sémiologie, au structuralisme de Prague à la psychologie cognitive et aux modèles d'action et de communication des sciences sociales, on peut ramener cette affirmation à sa substance de base en disant que la production et la réception de textes médiatiques doivent être interprétées comme schéma d'action et d'expérience. La théorie et l'histoire des média devront donc tenir compte de l'aspect social de ces schémas d'action et de leurs fonctions ${ }^{2}$. Il en résulte des différences dans la recherche et la méthodologie sur les média selon l'approche historique ou théorique. Si la recherche théorique a pour objectif la reconstruction de schémas d'action généraux et abstraits (mentaux aussi naturellement), la pragmatique historique, elle, s'emploie à déterminer et à décrire la fonction de schémas spécifiques d'action médiatique dans un contexte social et historique. Nous allons d'abord dégager les principes de base de la pragmatique historique du texte pour pouvoir les utiliser ensuite comme cadre de référence à quelques thèses visant à développer une pragmatique historique du film. Cela nous servira aussi, d'une part, à vérifier la validité des modèles textuels en ce qui concerne la recherche sur le cinéma et la télévision et, d'autre part, à faire une première proposition, sur une base pragmatique, de l'analyse des schémas d'action concernant les «films d'auteurs» de la Nouvelle Vague.

\section{Pragmatique historique du texte et histoire de la fonction}

Se basant sur «l'esthétique de la réception ${ }^{3}$ » de Hans Robert Jauss, tout en tenant compte de la théorie de l'acte de locution élaborée par Austin et Searle 4 et de la phénoménologie d'Alfred Schüt ${ }^{5}$, Karlheinz Stierle propose de faire du concept d'action la catégorie centrale des sciences de la littérature ${ }^{6}$. Alors qu'Austin et Searle se limitent à l'acte de locution singulier, Stierle va plus loin en intégrant cet acte de locution dans une théorie générale de l'action. Le postulat théorique selon lequel le texte est action est 
riche en conséquences pour les sciences du texte. On ne peut plus définir les catégories de genres et les genres littéraires par des entités ontologiques (poésie, prose, drame, etc.). Au contraire, ils se révèlent être des modèles (typiques) d'actions ou d'actes de locution dans des contextes historiques spécifiques. Les sciences et la théorie du texte doivent alors se situer dans le cadre des sciences de l'action. Ce qui fournit les conditions requises pour le développement d'un modèle d'une histoire de la fonction, permettant d'examiner l'interaction entre l'auteur, le texte et le lecteur dans des situations historiques de communication données. Avec sa pragmatique historique du texte, Hans Ulrich Gumbrecht a développé un modèle de texte - aussi basé sur la sociologie de la connaissance - qui prend pour objet central les schémas d'expérience et d'action, concrets et historiques de l'auteur et du lecteur ${ }^{7}$. Ce modèle d' interprétation sert de base à la reconstruction de motifs de la production et de la réception des textes et à la reconstruction des schémas spécifiques d'expérience des lecteurs. Autrement dit, ce modèle reconstruit les schémas d'action spécifiques de textes (fictifs et non fictifs) et nous mène à une histoire de la fonction. La position dans les différents contextes historiques s'avère être d'une importance capitale pour l'analyse du texte pour ce qui est de la détermination et de la description de sa fonction.

Voyons maintenant quels sont les principaux constituants du modèle de la pragmatique historique et comment on peut définir leur interaction. La réception et la production de textes s'opèrent dans des situations spécifiques de communication. La situation délimite l'horizon, au sens phénoménologique, de l'action verbale, initié par le médium «texte». Dans sa totalité, cette situation ne peut être déterminée, voire même définie de manière restrictive. Malgré le caractère, ouvert par principe, des situations et des difficultés théoriques et «pragmatiques» qui en découlent quand il s'agit de saisir dans leur totalité les cadres situatifs, nous pouvons thématiser certains facteurs de l'horizon situatif et examiner leur validité en ce qui concerne l'action textuelle. Ces facteurs sont la condition nécessaire au déploiement de la fonction sociale et historique des textes, et la reconstruction de ces cadres permettra d'émettre quelques hypothèses. Pour ce qui est de 
l'horizon situatif, on peut relever les éléments suivants: les conditions de base spatio-temporelles de toute action humaine ${ }^{8}$, les données et processus spécifiques de l'histoire mentale et sociale, le stock historique et social de savoir ${ }^{9}$, la langue à laquelle revient un rôle priviligié dans la construction sociale de la réalité et les créances ${ }^{10}$ (c'est-à-dire des éléments non-questionnés de savoir chargés de réguler notre comportement et nos actions et que nous considérons comme «vrais»). La reconstruction des différents éléments du stock social de savoir et du stock subjectif de savoir du lecteur, auxquels renvoient les textes et auxquels ils se réfèrent, permet de délimiter la fonction historique des textes et de formuler des hypothèses sur cette fonction. La situation de communication constitue le cadre historique dans lequel les actions entre l'auteur, le texte et le lecteur évoluent. On peut qualifier les différents processus spécifiques entre l'auteur, le texte et le lecteur comme situation de communication «interne» (fictive ou non).

Venons-en maintenant à d'autres constituants de la pragmatique historique du texte: l'auteur se trouve dans un contexte historique et social particulier qu'il «filtre» et interprète grâce à son stock subjectif de savoir. Ce dernier dépend du stock social de savoir de sa situation historique (nous ne disposons que d'une part restreinte de ce qui peut être su). Par le biais de processus d'expérience, ce savoir lui permet de constituer certains motifs d'action et certaines esquisses d'action, passant toujours par l'idée d'un lecteur prospectif. Quand il transpose la fonction intentionnelle de son texte en action littéraire, l'auteur doit choisir son médium, son genre. Les différents modèles de genres impliquent différentes possibilités en ce qui concerne l'amplitude de l'action entre l'auteur, le texte et le lecteur. La production de texte n'est pas la «voie à sens unique» de la réalisation linéaire et consciente des motifs d'action de l'auteur. Au contraire, les textes développent un dynamisme qui leur est propre et renvoient aux motifs premiers de la production de textes. Dans ce sens, «l'auteur» est une construction intentionnelle, une représentation et une idée qui, dans «l'acte créateur» de la production de textes, disparaît derrière le texte.

Les textes ont des structures propres à leur genre et s'inscrivent toujours dans une tradition marquée par l'histoire de la 
fonction qui se base sur l'attente du lecteur et sur l'horizon de cette attente. Leur répertoire joue avec les éléments du savoir social et historique, ils renvoient aux situations historiques, aux mentalités et aux «questions sans réponses» des sociétés. Comme l'auteur, le lecteur dispose d'un stock spécifique et historique (subjectif) de savoir qui fournit le support à ses propres motifs de réception du texte de même qu'à la fonction du texte qu'il réalise lui-même.

Dans le cadre scientifique de la pragmatique historique du texte, le lecteur/récepteur ne peut que dans une certaine mesure rester pure construction ${ }^{11}$. Même si, grâce aux méthodes empiriques de la recherche sociale, nous sommes à même, pour ce qui est du lecteur actuel, de reconstruire des éléments de son point de vue, de ses motifs et de son savoir social, cela ne peut rendre compte que d'une étroite tranche de son stock subjectif de savoir, limitant ainsi les résultats de l'analyse de la réception. Cet état de fait a encore plus de conséquences quand il s'agit de la reconstruction d'un lecteur historique d'un certain texte. En ce qui concerne la fonction sociale du texte, notre recherche sur les mentalités et nos reconstructions nous permettent seulement de formuler des hypothèses se rapportant à des domaines étroits et clairement délimités. La plausibilité de telles hypothèses reste à discuter. Cette lacune méthodologique de l'approche pragmatique ne devrait pas empêcher le chercheur de s'interroger sur le «ce que ça nous fait»" ${ }^{12}$. C'est ainsi que le processus décrivant la manière dont les textes ont pu ou auraient pu toucher le lecteur historique et la manière dont ils nous touchent aujourd'hui se trouve au centre des préoccupations scientifiques. En recherchant des réponses à ces questions, on pourra rendre compte des différences entre la situation historique de communication des époques révolues et notre situation actuelle de communication et montrer des aspects de l'évolution de la fonction du texte.

L'esquisse de ce modèle pragmatique permet, sur le plan de l'histoire sociale et celle des mentalités, de limiter la fonction historique des textes et des genres spécifiques. Et même si ce modèle ne fournit pas de résultats objectifs et même s'il réduit le savoir social à des domaines clairement délimités, il faut reconnaître qu'il dispose d'un avantage de taille: il peut générer des résultats de façon à la fois contrôlée et intelligible, et il s'agit 
maintenant de discuter la plausibilité de tels résultats. La démarche de la méthode «structurelle-fonctionnelle-structurelle» contribue largement à la transparence de la pragmatique historique du texte. Cette méthode peut être caractérisée de la manière suivante:

a) dans un premier temps, après une première lecture de repérage du texte à interpréter, on émet une hypothèse sur l'élaboration du sens envisagée chez le lecteur et sur la fonction intentionnelle du texte spécifique ${ }^{13}$. La formulation de cette hypothèse sur la fonction suppose la connaissance des processus historiques et littéraires qui constituent l'horizon de la production et de la réception du texte.

b) Au cours de la deuxième étape, celle de l'analyse de la structure, on examine tous les phénomènes du texte pour vérifier s'ils concordent ou non avec l'hypothèse émise. Il en découlera donc une conformité ou une non-conformité à l'hypothèse de départ, ce qui conduira à ...

c) une révision, une confirmation ou une précision de l'hypothèse sur la fonction.

En raison de ce que cette méthode exige de l'historien (qui en effet pourrait prétendre connaître tous les processus impliqués dans les interactions entre l'auteur, le texte et le lecteur?) seules, certaines parts des actions textuelles peuvent entrer dans le collimateur du chercheur. Cette réduction, une des conditions centrales de la recherche scientifique, encourage le chercheur à limiter théoriquement et méthodologiquement les perspectives et l'objet de ses recherches. Ce qui nous amène à la question de savoir si l'esquisse d'un modèle pragmatique du texte peut être utilisé dans l'analyse du film et des média.

\section{Pragmatique historique et cinéma}

La communication audiovisuelle peut être envisagée, tout comme la communication textuelle, comme schéma d'action en situation historique. Les textes écrits et les «textes» audiovisuels déploient leur fonction sur un arrière-plan de structures de savoir et de mentalités historiques. À première vue, notre modèle élaboré dans un contexte textuel peut sans difficulté s'adapter à l'analyse des films et des média. En revanche, après un examen plus approfondi, des divergences importantes apparaissent. 
$\mathrm{Si}$, dans le cas de la communication textuelle, il avait été question d'une intention del'auteur, c'est-à-dire d'une hypothèse sur la fonction étant alors une construction et une supposition scientifiques qui n'avait pas besoin de coïncider avec l'intention explicite de l'auteur ou d'un «sujet créateur», ceci est encore plus vrai dans le cas de l'analyse de la communication filmique. En effet, un entrelacs dynamique et interférent de chaînes d'action est à l'origine de la production cinématographique, et on ne peut pas leur attribuer à chaque fois une seule intention dominante. Le contenu et la structure de l'ultime texte médiatique (un film après le montage par exemple) sont profondément déterminés par une suite de réceptions et de modifications qui sont venues entrecouper le processus de production, ce que la production de textes littéraires ne connaît point. Il se produit un grand nombre d'interactions et de transformations qui, par exemple, dans le Cinéma de Qualité, c'est-à-dire le cinéma d'après-guerre, précurseur de la Nouvelle Vague, vont du modèle littéraire au montage final du film, en passant par le scénario et la mise en scène du réalisateur et de son équipe. Ces modifications comprennent non seulement l'interprétation et la modification des motifs d'action originaux mais aussi la transposition intermédiatique de suites de textes en suites d'images et de sons ${ }^{14}$.

À la différence de la communication textuelle, les processus de production de la communication filmique s'avèrent être de très complexes systèmes d'action qui lient étroitement la production et les modèles spécifiques de réception. Au cours de la deuxième phase de la production au plus tard, l'organisation et la coordination d'intentions et d'actions, le travail d'équipe, les procédures techniques et les facteurs institutionnels et matériels jouent un rôle prédominant. L'acte de production cinématographique vise généralement un cercle de récepteurs clairement délimité et défini et comprend les formes les plus diverses de feedback.

Il convient aussi de savoir qu'une pragmatique historique du film doit tenir compte du caractère spécifique des signes et du code spécifique de la production cinématographique. J'utilise le concept de texte médiatique au sens métaphorique, sans vouloir déboucher sur l'implication (osée?) d'un parallèle, voire même d'une identité, entre la langue ou le texte et le produit audiovisuel. 
Il me semble néanmoins utile de conserver ce concept de texte médiatique dans la mesure où il renvoie au caractère structuré du film et aux processus d'élaboration du sens chez le spectateur qui viennent s'y rattacher. Pour ce qui est du code filmique, la sémiologie et la sémiotique affichent de bons résultats et présentent de bonnes méthodes d'analyse qui pourraient être utilisées dans les trois étapes de la méthode «fonctionnelle-structurellefonctionnelle». Tout particulièrement en ce qui concerne la composition de l'image, les formes de montage et les structures narratives, etc.

On pourra, sur la base d'une pragmatique historique du cinéma, comme dans la communication textuelle, saisir et décrire les genres en tant que systèmes d'action, systèmes typiques ${ }^{15}$ etmédiatiques. Les modifications de différents éléments du système apportent automatiquement une modification de la fonction de tel ou tel film situé dans la tradition d'un certain genre ${ }^{16}$. Une pragmatique historique du film dirige l'attention du chercheur sur les déplacements et les modifications de la réception et des processus d'élaboration du sens - sur un plan à la fois synchronique et diachronique. Elle est un outil qui permet de dégager les différents modèles de réception des films et des textes médiatiques au sein de différents contextes institutionnels et de différentes situations historiques ${ }^{17}$.

En somme, la théorie et les méthodes de la pragmatique historique du texte peuvent être transposées dans le domaine des média audiovisuels. On ne peut toutefois pas reprendre tel quel le modèle d'action de la pragmatique historique du texte et l'appliquer à l'analyse des films et des produits audiovisuels. Il faut lui apporter - surtout en ce qui concerne la production cinématographique - de nombreuses modifications. Il faut également tenir compte du fait que nous ne devons pas partir d'un dialogue à dimension unique entre l'auteur (voire même «l'émetteur») et le spectateur (ou le «récepteur»), mais au contraire d'un système d'action aux dimensions multiples. Néanmoins une telle pragmatique du film semble offrir un instrument prometteur pour la reconstruction et l'analyse de la fonction historique de textes médiatiques. Je voudrais maintenant démontrer rapidement sa validité par une analyse paradigmatique de la Nouvelle Vague, de 
la notion d'auteur en tant que motif principal de l'action médiatique et par une brève analyse d'À bout de souffle de Godard, un filmculte de la Nouvelle Vague. Notre analyse se penchera sur la question de savoir si et dans quelle mesure on peut trouver dans un contexte national et discursif différent, celui des Pays-Bas des années 50 et 60 , des indications sur le schéma d'action intentionnel de la «notion d'auteur». Nous utiliserons un corpus de 17 critiques consacrées à $A$ Bout de Souffle et publiées en 1960 dans différentes revues néerlandaises.

\section{La Nouvelle Vague, action médiatique et innovatrice}

La Nouvelle Vague, c'est une invention de journalistes... Ce que font les nouveaux cinéastes, je voulais le faire en 1937.

Pierre Melville ${ }^{18}$

Le terme de la Nouvelle Vague ${ }^{19}$ marque dans l'histoire européenne du cinéma une des rares phases au cours desquelles le débat critique sur le cinéma coïncide (apparemment?) avec la réalisation, dans la production et la réception cinématographiques, des postulats esthétiques de ce débat. Les thèses de Bazin et de Truffaut publiées dans les Cahiers du cinéma et la présentation de centaines de nouveaux films dans les ciné-clubs parisiens ${ }^{20}$ renvoient à un système d'action médiatique apparemment idéal pour une analyse basée sur la théorie de la fonction.

En France, au cours des années 50, le système filmique d'action a connu une véritable poussée innovatrice dont les effets se ressentent encore aujourd'hui. Le cinéma de la Nouvelle Vague et ses concepts esthétiques et fonctionnels sont devenus des modèles, à la fois pour des formes particulières de la production, pour la réalisation dans une œuvre de l'intention spécifique et subjective d'un «créateur» et enfin pour les modèles de réception qui se rattachent au film d'art. Comme tout modèle, ou toute image historique ${ }^{21}$, le schéma d'action Nouvelle Vague se distingue dans une large mesure par des constructions imag(e)inées ou mentales. En d'autres termes, on ne peut définir la particularité de la Nouvelle Vague en répertoriant les soi-disant caractéristiques d'une série de films français qui furent tournés entre 1958 et $1962^{22}$, alignant ainsi jump cuts, shaky hand-held camera, disruptive editing, etc. ${ }^{23}$, mais par des schémas spécifiques d'ac- 
tion reposant principalement sur des représentations basées sur l'histoire des mentalités et sur des éléments de savoir se rapportant au cinéma et à sa fonction sociale et esthétique ${ }^{24}$. Le concept de la Nouvelle Vague présuppose la connaissance de cette imag(e)ination historique et de cette configuration de savoir de la part des producteurs et des récepteurs.

\section{Notion d'auteur et fonction historique}

La notion d'auteur suppose qu'il existe l'art du film et son contraire.

Georges Sadoul ${ }^{25}$

On peut dire que chaque film nouvelle vague est une crise d'épilepsie de la part de son metteur en scène.

Michel Fabre ${ }^{26}$

Lorsque, en 1954, François Truffaut exigeait un revirement radical du cinéma français dans «Une certaine tendance du cinéma français», cet article aujourd'hui célèbre marquait le début théorique de la Nouvelle Vague. Ses critiques à l'égard de «la tradition de qualité», tradition qui n'avait pas su s'émanciper de leurs modèles littéraires, et son playdoyer pour un cinéma d'auteur ${ }^{27}$ étaient au cœur du débat sur la fonction sociale et esthétique que l'éditeur et les auteurs des Cahiers du cinéma menaient depuis le début des années 50. «La politique des auteurs» de Bazin et les critiques positives de films (ceux de Renoir par exemple) montrant clairement les traces d'une paternité subjective, et la «caméra stylo», concept cher à Astruc, avaient ouvert la voie à la renaissance de la notion d'auteur ${ }^{28}$. La production cinématographique revenait alors à l'acte créateur, d'un individu, d'un sujet créateur, comme, quelques années plus tard, les premiers films de la Nouvelle Vague ont semblé le suggérer. La réception et la critique cinématographiques des années 50 et du début des années 60 ont tenu compte de ce fait.

Si nous considérons l'historiographie de la Nouvelle Vague, il apparaît alors qu'intentions subjectives et créatives d'action, textes filmiques, possibilités de réaliser l'intention dans l'acte de réception, sont étroitement entremêlés et que cette imbrication est prise telle quelle, considérée comme un fait accompli. Si nous acceptions ce point de vue, nous pourrions immédiatement clore 
notre étude pragmatique en déclarant que le système médiatique (et innovateur?) du système de communication de la Nouvelle Vague se caractérise apparemment par une reprise des schémas de la communication littéraire et des modèles de fonction correspondants. Mais cette conclusion rapide néglige un aspect important, à savoir que le modèle d'auteur élaboré par Truffaut et ses amis ne devait pas être compris comme description des processus de communication de la production et de la réception cinématographiques mais au contraire comme stratégie spécifique, comme configuration historique de savoir qu'il convient de situer dans un contexte basé sur l'histoire des mentalités et qui permet d'ouvrir des espaces spécifiques de fonction à la réception cinématographique.

On peut reconstruire des motifs visant à démontrer pourquoi la théorie et la pratique cinématographiques des années 50 ont apporté cette signification au concept d'auteur, 10 ans avant que Roland Barthes n'en postule la mort ${ }^{29}$, une signification qui a cours aujourd'hui encore, du moins dans le cinéma américano-européen. Ainsi l'auteur de la Nouvelle Vague ne doit pas être envisagé comme l'instance émettrice traditionnelle d'un système de communication sciemment linéaire, aboutissant au récepteur, mais comme la construction sociale et historique d'éléments de savoir. Cette construction est en corrélation avec les besoins spécifiques, latents même d'un groupe socia1 et elle est accessible à l'analyse pragmatique. Cette fiction d'un sujet, ou d'un auteur derrière le film, a longtemps voilé la perception des processus et des transformations intermédiatiques, s'opérant entre le modèle textuel, le scénario et la réalisation filmique. Elle a longtemps encouragé le spectateur à rechercher les indices révélant la subjectivité et la créativité de l'auteur dans le texte filmique.

Je vais dans ce qui suit dégager quelques motifs ${ }^{30}$ pour déterminer le syndrome intentionnel de savoir de la notion d'auteur. Je vais donc me demander quels éléments de savoir historique il faut faire ressurgir pour construire cette idée d'auteur et de créateur et quelles sont les fonctions historiques que l'on peut rattacher à ce concept. Comme des exemples de réception de ce débat ont déjà été publiés dans le milieu francophone ${ }^{31}$, il me paraît intéressant de se demander, dans une rapide analyse des critiques néerlandai- 
ses d'À bout de souffle, dans quelle mesure des éléments du concept d'auteur ont pu être perçus et réalisés dans un contexte culturel différent en interaction avec la réception du film. La section sept de cet article, intitulée $\grave{A}$ bout de souffle - concept d'auteur de la Nouvelle Vague et sa réception aux Pays-Bas, rendra compte de cette analyse. Concernant la méthode basée sur l'histoire de la fonction, il convient de mentionner que la deuxième étape (analyse structurelle) restera absente du «contexte pragmatique» de cet article. Il serait certainement très prometteur d'examiner dans une vaste étude pragmatique un important corpus de films de la Nouvelle Vague pour déceler des indices et des traces de la notion d'auteur et d'analyser leur importance pour le système de communication et d'action de ce courant. Toutefois, la longueur et le cadre restreint de cet article justifient le fait de ne soumettre le concept pragmatique de la notion d'auteur qu'à l'analyse heuristique d'un corpus littéraire de 17 critiques cinématographiques néerlandaises et non pas des textes filmiques.

\section{L'auteur - construction de la Nouvelle Vague basée sur une mentalité et sur un savoir historiques}

Alors que dans le débat postmoderne, l'auteur, en tant qu'instance, n'est plus derrière son œuvre, présent tout au plus comme le scripteur d'un texte placé à l'extérieur de lui-même, de sa propre autorité, cet auteur connaît sa grande et dernière renaissance par et dans un médium dont les schémas d'action abandonnent, excluent même d'emblée le concept individuel d'auteur. La production et la réception cinématographiques ne comprennent pas les réalisations linéaires des intentions d'action isolées et subjectives d'un seul réalisateur, d'un seul créateur. Faire et regarder un film est toujours un jeu intertextuel, intermédiatique des intentionnalités qui se surchargent, s'entrecoupent et vont même jusqu'à se concurrencer. C'est aussi le cas quand réalisateur et scénariste sont une seule et même personne. Au cours des années 50, les «théoriciens» et les «praticiens» de la Nouvelle Vague connaissaient bien ce fait et pourtant, ils ont décidé de donner, dans les films, une place centrale à la notion d'auteur, à «la politique des auteurs». Le fait que la notion d'auteur de la Nouvelle Vague et de ses protagonistes n'ait pas été élaborée sous 
forme d'une théorie cohérente semble apporter un élément à notre thèse, selon laquelle il faut voir dans cette configuration historique de savoir une construction mentale qui, en tant que concept, en tant qu'idée, a offert à ce cinéma jeune et nouveau une série de modèles de fonction. Mais quels sont les éventuels «contenus» du «syndrome d'auteur» de la Nouvelle Vague et quelles sont les relations entre les motifs qui les caractérisent ${ }^{32}$ et enfin quelles possibilités de fonction découlent de cette configuration de savoir?

Je vais maintenant avancer quelques thèses sur les implications qu'entraîne la notion d'auteur pour les structures communicatives de l'action filmique de la Nouvelle Vague. Ces thèses qui ne sauraient prétendre à l'exhaustivité dans la description de toutes les dimensions du syndrome d'auteur, présentent des reconstructions «scientifiquement contrôlées» d'une configuration de savoir et s'étendent à partir d'un point de vue concret et apparent, celui du cinéma et de la politique de marché, jusqu'aux domaines latents et dissimulés des aspirations et des motifs préet inconscients des producteurs et des récepteurs.

\section{L'auteur - une stratégie politico-médiatique}

Parler de l'auteur et de la notion d'auteur comme d'une stratégie politico-médiatique revient à opposer l'idée d'une expression subjective et l'idée d'étroites relations entre le créateur et son «ฉuvre» aux mécanismes économiques qui dominent le marché du film.

Et même si la plupart des jeunes réalisateurs ne pouvaient pas échapper complètement aux contraintes du marché et si leurs films ne pouvaient être produits que dans de grandes difficultés financières, la construction de la notion d'auteur leur permettait de se constituer un espace imaginaire à l'intérieur duquel ils étaient libres de créer leurs œuvres ${ }^{33}$. À cela correspond, du côté du public, un des modèles possibles de réception, le plaisir de reconnaître cette subjectivité et d'y participer. La «politique des auteurs» implique une délimitation et une singularisation dont les connotations positives prédominent: à propos du personnage d'un créateur que l'on peut imaginer et qui se distingue de la grande masse des artisans médiatiques; à propos de l'œuvre qui reçoit son statut «d'unicité» sur un fond d'une grande masse de films qui ne 
savent que répondre aux lois profanes et aux besoins du marché de masse; à propos du spectateur qui peut ainsi participer à une œuvre d'art et vivre une expérience artistique, cette dernière tente de retrouver le reflet d'une aura ${ }^{34}$ et promet au récepteur d'échapper à l'époque profane de la reproductibilité technique.

\section{Auteur - mémoire individuelle et collective}

Signer un texte en tant qu'auteur revient à se distinguer, par cet acte, de la masse d'intertextes anonymes, parallèlement présents - et ceci avant toute désignation d'activité créatrice.

Ce qui est marqué d'un nom peut, en raison de l'attribution du nom, plus facilement s' intégrer aux structures de validité ${ }^{35}$ du savoir social et être plus rapidement disponible. C'est donc la raison pour laquelle se faire un nom, signifie, aussi pour les auteurs de la Nouvelle Vague, se servir de ces structures du conscient pour pouvoir s'inscrire avec plus d'efficacité dans le système historique du savoir social. Le désir de durée de celui qui donne son nom correspond aux besoins du récepteur de réduire la complexité. Le nom de nombreux auteurs de films devient un véhicule mnémotechnique, un aide-mémoire basé sur une identité cohérente.

\section{Auteur et identité construite}

L'identité construite d'un auteur est l'instance qui "garantit» l'unité de l'œuvre. Elle permet de relier l'hétérogénéité des textes à l'identité personnelle d'une instance.

Ceci est vrai, tout aussi bien pour le producteur qui cherche à se réaliser dans son œuvre que pour le spectateur qui peut retrouver ce dernier dans le film. Le «style» devient alors une forme variable et décelable de l'expression d'un sujet auquel il donne des contours plus précis. Le concept de «l'identité» de l'auteur de la Nouvelle Vague a l'avantage de permettre de relier les questions sans réponses sur l'œuvre, les formes nouvelles et surprenantes du style, utilisées sciemment ou non par l'auteur, comme par exemple le principe bien connu des jump cuts, aux modèles d'expérience et aux intentions d'une instance perçue comme unité. C'est en ce sens que le concept d'auteur, dans un rapport d'action entre le spectateur et le film, permet de relever des effets inattendus et des éléments en situation de distanciation à partir du modèle 
traditionnel de perception esthétique: tout ce qui est hétérogène, fragmenté, en situation de distanciation et en rupture de cohérence est replacé, grâce à l'élément de savoir et au catalyseur de l'identité de l'auteur, sur un terrain plus familier, celui d'une perception esthétique intégrante.

\section{«Créateur» - génie et pose}

La pose de "créateur» ou de "génie» que prend le réalisateur déguise un texte médiatique dépendant des lois économiques $d u$ marché en un acte créateur unique.

Cet aspect de la configuration de savoir de la notion d'auteur rapproche le schéma d'action et de communication de la Nouvelle Vague de l'esthétique traditionnelle (bourgeoise) du génie, au $\mathrm{XIX}^{\mathrm{e}}$ siècle. Le créateur, le génie, la pose prennent la place pour une œuvre qui offre une jouissance esthétique et fait éclater les limites de l'environnement profane du récepteur. La pose du créateur et du génie solitaire, avec toutes ses implications, souligne le besoin du jeune réalisateur de se distinguer de la masse des tâcherons traditionnels du film. Quelques années après la fin de la Nouvelle Vague, Godard a transposé le syndrome du créateur dans la métaphore médiatique de son vidéo-film Scénario du Film Passion. Le créateur, posant entre le projecteur et l'écran, nous tournant le dos pendant que les images qu'il commente sont projetées sur l'écran et sur son $\operatorname{dos}^{36}$, marque la fin provisoire à cette évolution qui avait depuis longtemps déjà soumis la pose originale porteuse de sens (que les films de la Nouvelle Vague avaient déjà fragmentée) à une rupture intermédiatique et intertextuelle.

Au cours des années 50 , le modèle du créateur et du génie offrait aussi la possibilité d'associer le réalisateur à une version triviale de la philosophie existentielle de provenance sartrienne. Le sujet seul au monde et son ultime responsabilité de ses actes correspondent trop bien aux ébauches de l'identité personnelle des réalisateurs et aux «images d'identité» des protagonistes de leurs films.

\section{Auteur de films et action littéraire}

La production textuelle signifie un schéma dynamique et dense 
d'action entre l'auteur et le texte littéraire, qui, une fois achevé, fait disparaître l'auteur derrière son texte ${ }^{37}$. Mais elle offre en même temps, dans le processus d'écriture de cette instance, des possibilités immédiates ou médiates d'accès. En raison de conditions spécifiques et médiatiques, cet accès immédiat est impossible au cinéma. Si, par le biais du concept d'auteur, on peut rapprocher la production cinématographique de la production littéraire, on tentera ensuite de nier les différences spécifiques de ces deux média.

Quel pourrait être le gain pour le créateur, le théoricien, le spectateur de la Nouvelle Vague de cette négation (consciente?) des schémas spécifiques et médiatiques d'action? C'est évident, le film pourrait bien se voir attribuer ainsi les qualités esthétiques d'un autre médium esthétique reconnu. L'auteur de films sert d'instance de garantie pouvant utiliser, pour un médium différent, un type particulier et reconnu d'action sociale doté de connotations culturelles positives (écrire de la littérature et lire). La construction d'auteur de la Nouvelle Vague poursuit à sa manière un débat qui remonte jusqu'à l'émancipation du cinéma du contexte vaudevillesque. Des motifs reconnus dotés de connotations positives servent d'instance de légitimation pour la valeur esthétique de la production et de la réception cinématographiques; ces modèles réducteurs créent une base culturelle commune entre le réalisateur, le spectateur et la critique cinématographique.

Quel rôle revient à ces configurations de savoir dans un contexte socialement différent, celui des Pays-Bas de la fin des années 50 et du début des années 60 ? C'est ce que je vais reconstruire par l'analyse des critiques néerlandaises du film de Godard $\grave{A}$ bout de souffle.

\section{7. À bout de souffle - concept d'auteur de la Nouvelle Vague et sa réception aux Pays-Bas ${ }^{38}$}

À bout de souffle porte, comme une œuvre littéraire, la marque de son auteur et est même partiellement autobiographique ${ }^{39}$.

Avant de commencer l'analyse du contenu des critiques néerlandaises d'À bout de souffle, j'aimerais faire quelques remarques sur le statut du matériel de base et sur la méthodologie adoptée. Il faudra, comme dans toute étude de réception reposant sur un 
matériel de départ composé de textes écrits, tenir compte des faits suivants:

a) ces textes - nos critiques cinématographiques aussi représentent des reconstructions d'expérience et de réception, qui développent des modèles d'interprétation basés sur des structures cognitives spécifiques et sur des éléments historiques de savoir;

b) générant une cohérence, les critiques servent de véhicule et permettent d'enrober un ou plusiers textes médiatiques qui nécessiteraient une explication dans une situation institutionnalisée et communicative ${ }^{40}$;

c) ces textes comprennent des processus de transformation intermédiatiques;

d) ils impliquent des actualisations spécifiques d'éléments appartenant au domaine du savoir social — ou si nous préférons d'éléments des idéologies dominantes ${ }^{41}$ — pour pouvoir exercer leur fonction référentielle;

e) les critiques cinématographiques font partie de traditions spécifiques de genre, qui se distinguent par des schémas d'action et de communications spécifiques et historiquement variables (en France le débat sur la Nouvelle Vague en est un excellent exemple). Les relations entre ce débat et celui des textes médiatiques sont soumis à une évolution historique ${ }^{42}$.

Malgré la perte de son immédiateté, les critiques cinématographiques sont tout à fait appropriées pour les analyses de réception ayant pour objectif de connaître les configurations dominantes de savoir, qui manœuvrent les processus de la constitution du sens au cours de la réception d'un texte médiatique. En ce qui concerne ma démarche méthodologique, je voudrais également faire remarquer qu'en raison de l'étendue limitée des textes de base, une analyse qualitative du contenu semblait la plus adéquate. Elle a aussi l'avantage de cerner les cinq aspects du syndrome du savoir de la notion d'auteur qui viennent d'être présentés. Je renonce à décrire explicitement le contexte idéologique et politique des différentes revues; il ressort en général assez rapidement du contenu et des structures des critiques. L'analyse des contenus n'a été entreprise qu'après l'interprétation des catégories de «l'auteur» de la Nouvelle Vague, basées sur l'histoire des mentalités et préalablement élaborées. 
Je me permets enfin d'ajouter une courte remarque sur la construction de la notion d'auteur et sur son caractère national ou international. Nous avons supposé qu'aux Pays-Bas, en raison d'une situation sociale différente au sein de l'histoire des mentalités, le débat sur la Nouvelle Vague et ses auteurs se distinguerait assez nettement du débat français. Si nous considérons l'ensemble des critiques, cela est certes vrai pour quelques nuances spécifiques et locales (le rôle de l'Église par exemple ou la fonction de la scène cinématographique locale, celle des salles de cinéma). Cependant, le débat néerlandais ne fait que reprendre de nombreux contenus et sujets du débat français d'origine (publié dans les Cahiers du cinéma et autres revues). Cela est vrai en ce qui concerne le rôle et la fonction de l'auteur et certains autres aspects comme, par exemple, celui de la «teneur morale» d' $\grave{A}$ bout de souffle. À ce sujet, les critiques de tendance catholique mentionnent explicitement la conférence des évêques français qui s'est déroulée à Paris au printemps 1960. C'est avec grand plaisir que Cor de Groot cite dans son article «Nieuwe Golf zet door» (Volkskrant, 2 avril 1960) les commentaires sur la Nouvelle Vague que les évêques français avaient présentés lors d'une conférence de presse exprimant ainsi leur inquiétude au sujet du cinéma français. Les évêques soulignent que ces nouveaux films:

(...) sont un persiflage des vertus de la famille et des valeurs humaines, qu'ils attirent la cupidité et qu'ils instaurent des dérapages sexuels et le mépris de toute autorité.

On peut donc en accord avec la position française déconseiller au public d'aller voir le film. On peut expliquer le fait que la critique néerlandaise s'appuie sur la critique française par le décalage temporel de deux ou trois mois. À bout de souffle est en effet sorti aux Pays-Bas en mai 1960, et il était bien naturel de reprendre les critiques françaises. Il serait certainement très intéressant d'analyser minutieusement cette reprise intertextuelle et internationale.

En ce qui concerne notre question sur la fonction du concept d'auteur, on retrouve ce syndrome de savoir dans presque tous les articles comme un des élements les plus importants pour la «référentiatisation» critique et la reconstruction d' $\grave{A}$ bout de 
souffle. Il apparaît également que la question de la notion d'auteur occupe une place centrale au cours de la première phase de l'histoire de la réception de notre film parce qu'on ne savait pas exactement quelle part revenait aux deux protagonistes connus de la Nouvelle Vague, Truffaut et Chabrol, dans le scénario et la production d'À bout de souffle. Mais revenons maintenant à notre question de départ concernant le concept d'auteur et la critique cinématographique. Considérant la fonction historique de ce concept dans la critique cinémâtographique néerlandaise, quels modèles de base peut-on reconstruire?

\section{Le concept «Godard» et la Nouvelle Vague}

Dans presque toutes les critiques (14 des 17 articles), le nom de Godard est associé à celui de Nouvelle Vague d'une manière qui montre que la Nouvelle Vague ne peut être définie à partir de structures spécifiques de textes. Le nom de l'auteur et le film sont alors les exemples typiques du schéma médiatique d'action de la Nouvelle Vague.

En France aussi on considère le film de Jean-Luc Godard comme le film le plus typique de cette «Nouvelle Vague» dont on parle avec tant d'éloges ${ }^{43}$.

L'alliance de l'œuvre et du nom place le film dans une situation spécifique de communication, à laquelle les critiques réagissent avec plus ou moins d'admiration, ou bien, dans les commentaires de caractère religieux, avec un refus non dissimulé. Dans de nombreux cas, le nom de l'auteur ne sert pas seulement d'instance de garantie pour établir un lien-biographique avec le créateur, il est aussi le symbole d'une «nouvelle morale», de la rébellion de la jeunesse d'après-guerre envers les normes sclérosées de la bourgeoisie établie. Les critiques n'ont pas utilisé le concept Godard dans la perspective de la vraie personne de l'auteur mais comme construction pour déboucher sur l'élaboration du sens par le biais du texte filmique. Le fait que les avis dans les articles sur l'âge et la nationalité de Godard soient très divergents semble révélateur. Ce Français $(N R C)$ ou Suisse français (Elsevier) ou encore Suisse (Algemeen Dagblad) a, en juin 1960, 24 ans (Volkskrant, 2 avril 1960) ou 26 ans (Volkskrant, 14 septembre 1960, Algemeen Dagblad) ou 29 ans (Haagse Post) ou encore 30 
ans (Elsevier). Le nom de Godard est associé à un contexte social et géographique particulier mais sans référence - malgré quelques rares tentatives - à une biographie particulière ${ }^{44}$. Il renvoie au contraire aux configurations de savoir, aux mentalités associées généralement aux individus appartenant à une certaine tranche d'âge et plus particulièrement au groupe social gravitant autour des Cahiers du cinéma, en France, à la fin des années 50. C'est la raison pour laquelle l'arrière-plan suisse de sa «vraie vie» n'a que très peu d'importance. Ce qui, dans les critiques néerlandaises, attribue au concept Godard et à sa place au sein de la Nouvelle Vague, leur fonction porteuse de sens, n'est certainement pas en dernier lieu le renvoi explicite au conflit des générations, à la rebellion esthétique et politique qui se greffe sur le nom de Godard.

\section{Godard — élément de la «mémoire collective»}

Il est étonnant de remarquer que de nombreux articles (9 des 17 articles) renvoient de manière plus ou moins explicite à la notion d'auteur multitentaculaire d'À bout de souffle. À ce propos, le film-culte de la Nouvelle Vague s'avère être un paradoxe qui en raison de sa dénomination formelle de produit d'une équipe de créateurs (Truffaut, Chabrol, Godard) devrait être exclu du cadre théorique du concept d'auteur de la Nouvelle Vague. Le fait que cela ne soit pas le cas — à quelques rares exceptions près ${ }^{45}$ — mais qu'au contraire, aux points textuels névralgiques des critiques, seul Godard soit cité comme instigateur et réalisateur du film, pourrait être un indice exprimant le besoin de réduction de complexité que ressentent les récepteurs (celui d'un type particulier également celui de la corporation des critiques de cinéma). Dès la première phase de l'histoire de sa réception, $\grave{A}$ bout de souffle est lié à une instance construite et subjective dotée d'intentions subjectives.

Le réalisateur dirige l'action du film rapidement (apparemment?) sans émotions. On a de temps en temps l'impression que Godard craint que ses images soient plus suggestives qu'il ne le voudrait ${ }^{46}$.

Aux Pays-Bas, le mythe Godard remonte aux années 60. Même si l'apport créatif de Truffaut pour le scénario et de Chabrol pour la réalisation avait pu l'endommager, les critiques savent parer à 
cette éventuelle érosion en disant que les noms des deux autres protagonistes de la Nouvelle Vague n'apparaissent au générique du film que pour des raisons publicitaires et ne diluent en aucun cas la notion d'auteur:

Pour ajouter de l'intérêt au film par des noms connus on annonce que François Truffaut (réalisateur des 400 coups) aécrit le scénario et que Chabrol (réalisateur des Cousins) a dirigé le tournage. Ceci porte préjudice au talent exceptionnel et manifeste de Jean-Luc Godard. Truffaut n'a rien fait de plus que de fournir les informations contenues dans un article de journal de quelques lignes, et Chabrol a associé son nom au film pour éviter à Godard les problèmes qu'il aurait pu avoir avec le puissant syndicat des techniciens du cinéma, ces derniers ne supportaient pas de voir travailler la jeune génération avec un seul cameraman et sans s'entourer d'une armée de techniciens et d'ouvriers ${ }^{47}$.

Le recours aux noms déjà connus ne fait qu'étayer la fonction

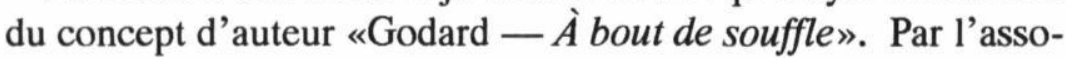
ciation avec Truffaut et Chabrol, le nom de Godard est plus facile à localiser et à mémoriser.

\section{Godard - art et style}

Rappelons-nous nos thèses concernant l'identité construite de l'auteur et la pose du créateur et nous pouvons retrouver cet aspect du concept d'auteur dans les différents articles néerlandais. Le génie, c'est-à-dire l'œil et la main de l'auteur, de Godard, (et non pas du cameraman Coutard) sont les instances intentionnelles auxquelles on attribue les remarquables particularités stylistiques d'À bout de souffle.

Godard apporte un sens neuf à ses «jump cuts» ce qui requiert une grande part de génie. Il semble supprimer des discussions d'une certaine longueur tout ce qui pourrait avoir une quelconque importance. D'autre part, il met dans la bouche de ses acteurs des dialogues dont le naturel fait peur ${ }^{48}$.

Le génie de Godard s'est inscrit dans ses textes filmiques. La question de savoir si les critiques expriment un avis favorable ou défavorable sur $\grave{A}$ bout de souffle n'a que peu d'importance par rapport à notre analyse de la réception; ce qui compte en revanche c'est, qu'aussi bien dans le camp des partisans, dans celui de ceux dont l'avis est plus mitigé que dans celui des adversaires déclarés, 
l'alliance est établie entre les caractéristiques structurelles spécifiques et filmiques d'une part et les intentions et constellations de motifs (comme modèles d'action) d'autre part. C'est la raison pour laquelle les mêmes caractéristiques d' $A$ bout de souffle peuvent aussi bien être comprises comme: libération de l'art du cinéma, éclatement conscient des traditions, instigatrices d'une «nouvelle tension nerveuse» (Groene Amsterdammer) ou comme ensemble d'effets surprenants qui débouchent sur un «jeu apprêté et surfait» (Volkskrant, 14 mai 1960). L'élément de savoir, actualisé dans tous les articles qui tiennent compte des processus spécifiques et innovateurs du film, est basé sur l'idée d'un créateur subjectif qui se réalise dans sa production (artistique?). Même dans sa forme dotée de valeurs négatives, le concept permet de surcroît d'associer «les procédés stylistiques impropres» et le contenu problématique d'À bout de souffle à une identité construite et subjective afin de pouvoir conférer à ce film le statut de document psychosocial de son époque, tout en lui refusant évidemment celui d'œuvre d'art (accordé au contraire à Un condamné à mort s'est échappé de Bresson, 1956). C'est ce qui se passe dans les critiques de tendance catholique. En raison de l'insouciance de l'auteur et de son film, $A$ bout de souffle ne devrait plus être joué dans les salles publiques:

(...) à interdire en raison d'une conception tout à fait nihiliste de la vie, exprimée par les acteurs principaux: on construit un drame dans lequel meurtre, vol et rapt sont ébauchés dans la plus grande insouciance, sans le moindre contrepoids moral et dans lequel l'absence de toute morale sexuelle est si largement étalée dans les mots et les images que ce film ne peut être projeté dans les salles publiques $^{49}$.

Ainsi non seulement son contenu, mais aussi son style ne permettent une projection publique. Ce sont pourtant d'un autre côté les éléments de style surprenants qui, en tant que signes artistiques, déterminent aux yeux des critiques la valeur esthétique du film. On ne les considère donc pas uniquement comme l'expression d'une exploitation maladroite du médium film, comme l'expression de l'œuvre enfantine d'un amateur, mais comme des éléments en situation de distanciation ${ }^{50}$, sciemment utilisés par l'instance Godard. 
Par des procédés modestes, il [Godard] a voulu lui [l'histoire] donner un air de sincérité, de vraisemblance et il a en grande partie réussi ${ }^{51}$.

Les procédés de style, les effets de distanciation, l'explosion des traditions de genres sont reliés aux intentions d'action de l'instance d'auteur constituées et construites par le critique et/ou le lecteur. C'est aussi vrai pour les procédés esthétiques du film documentaire que l'on peut y retrouver, comme celui de la caméra qui se déplace allant explorer de nouveaux espaces à l'extérieur des studios de cinéma et qui est dorénavant intégré au contexte des films de fiction.

Abordons en conclusion un autre aspect du syndrome d'auteur «Godard»: la pose du créateur et du génie, que prend l'auteur et qui se trouve esquissée et contenue dans le schéma d'action de la Nouvelle Vague, se heurte à la réprobation de nombreux critiques. «Godard est blasé», ce chronotope des critiques cinématographiques remonte aux Pays-Bas à son premier film $\dot{A}$ bout de souffle. L'usurpation du modèle du génie associé à l'usurpation du modèle de la communication littéraire ne fait pas automatiquement monter la cotation de la communication cinématographique et médiatique ainsi définie. En effet, en raison de la revendication qui lui est adjointe, ce schéma d'action de la communication littéraire ne peut que déboucher aussi sur des connotations négatives. Aux yeux des critiques, le metteur en scène doit, en tant qu'auteur, se mesurer au schéma d'action de la communication littéraire et peut facilement passer pour un «virtuose frimeur», un «snob» (Haagse Courant), un «amateur prétentieux», un «dilettante» (Parool) qui a crée «un produit surfait et apprêté» (Volkskrant, 16 mai 1960). Le concept d'auteur de la Nouvelle Vague n'est apparemment pas toujours capable d'honorer, dans le débat critique, ses propres exigences (à propos de la connotation positive du modèle, à laquelle il aspire). Ceci est vrai pour la critique néerlandaise comme pour la critique française et ce fait renvoie avec «ses variances et ses connotations négatives» à une fonctionnalisation historique et aux possibilités de fonction de la configuration de savoir de la notion d'auteur. 


\section{Pragmatique du cinéma - concept d'auteur et fonction historique}

L'analyse de la réception des critiques néerlandaises d' $\grave{A}$ bout de souffle a fourni de premiers indices concernant la valeur scientifique de notre ébauche d'une pragmatique historique du cinéma. $\mathrm{Si}$, à partir de ce concept théorique, nous pouvons affirmer que la production et la réception cinématographiques doivent être interprétées comme des systèmes multidimensionnels d'action et qu'en association avec ces systèmes, des configurations historiques de savoir ont une influence décisive sur les processus d'élaboration du sens tant pour le producteur que pour le récepteur, l'objectif principal de la recherche pragmatique sera alors de reconstruire la fonction historique, à savoir l'interaction des éléments d'un système ainsi défini. Les aspects des institutions médiatiques et du pouvoir médiatique (et politique?) ne sont pas exclus de ce système, mais bien au contraire, saisis comme éléments historiques de savoir et il reste à examiner leur effet sur l'histoire de la fonction et de la réception.

Sous cet angle, il ne faut pas voir le concept d'auteur de la Nouvelle Vague comme «l'intention d'un sujet historique» mais comme un syndrome de savoir basé sur l'histoire des mentalités et manœuvrant la production et la réception cinématographiques. Par conséquent, nous ne voyons pas dans l'auteur de la Nouvelle Vague le véritable acteur mais la construction de savoir social. Cette construction dirige, comme nous l'a montré l'analyse des critiques néerlandaises d'À bout de souffle, les processus de l'élaboration du sens pour les récepteurs, et ces derniers la perpétuent tout en la différenciant. Elle constitue une unité du «créateur et de l'œuvre» et englobe une série de transformations intertextuelles et intermédiatiques (allant du scénario à la critique cinématographique). En ce qui concerne l'analyse, sur une base pragmatique, de la réception des articles néerlandais, il apparaît clairement que le concept d'auteur joue un rôle prédominant pour la réception d'À bout de souffle (et certainement pour la réception de centaines d'autres films et pas seulement de la Nouvelle Vague). En outre, les résultats révèlent la nécessité de réviser le concept du cinéma national et des espaces internationaux de réception. La question se pose alors de savoir dans quelle mesure 
on peut encore parler de débat national au sein de la critique cinématographique (du moins depuis la Deuxième Guerre mondiale). Notre analyse a montré certains aspects spécifiques du contexte néerlandais et de la fonction de cette configuration historique de savoir (par exemple, le rôle assez influent de l'Église dans un paysage audiovisuel divisé en colonnes encore en $1960^{52}$ ). Mais en ce qui concerne la réalisation du concept d'auteur, nous n'avons établi aucune différence par rapport au fameux débat français. Aux Pays-Bas comme en France, l'usurpation du modèle de la communication littéraire semble servir l'élaboration du sens d'À bout de souffle. Une étude complète et comparative de la réception devrait pouvoir vérifier cette supposition.

Malgré les incertitudes, en ce qui concerne les rôles des coauteurs Truffaut et Chabrol, les contours du concept d'auteur «Godard» se précisent dès la première phase de l'histoire de la réception. Des analyses sur une base pragmatique de la réception pourraient aujourd'hui en examinant l'histoire ultérieure de la réception d'À bout de souffle et d'autres œuvres de Godard apporter des éclaircissements sur la concrétisation de ce concept. Une telle analyse serait sans doute à même d'établir des corrélations entre les caractéristiques structurelles d'À bout de souffle et les différentes élaborations de sens chez les récepteurs et d'écrire ainsi l'histoire de la fonction de ce film-culte de la Nouvelle Vague.

Université d'Amsterdam

\section{NOTES}

$1 \mathrm{Je}$ tiens à remercier vivement Mme Véronique Meyer, de Mannheim, pour son travail et son dévouement dans la traduction de cet article.

2 Cf. Jürgen E. Müller, «Literaturwissenschaftliche Rezeptions - und Handlungstheorien», dans. K.-M. Bogdal (éd.), Neue Literaturtheorien (Opladen: Westdeutscher Verlag, 1990) p. 176-201.

3 Hans Robert Jauss, Literaturgeschichte als Provokation der Literaturwissenschaft (Frankfurt a. M., 1970). 
4 John L. Austin, Zur Theorie der Sprechakte (Stuttgart, 1972). J. R. Searle, Speech Acts (Cambridge, 1969).

5 Alfred Schütz, Der sinnhafte Aufbau der sozialen Welt (Frankfurt a. M., 1974).

6 Karlheinz Stierle, Text als Handlung (München, 1975) surtout p. 9 et suivantes.

7 Hans Ulrich Gumbrecht, «Receptie esthetica en handelingstheoretische literatuurwetenschap", dans Charles Grivel (éd.), Methoden in de literatuurwetenschap (Muiderberg, 1978).

8 Àce sujet, cf. Alfred Schützet Thomas Luckmann, Strukturen der Lebenswelt (Neuwied/Darmstadt, 1975).

9 Cf. Peter Berger et Thomas Luckmann, Die gesellschaftliche Konstruktion der Wirklichkeit (Frankfurt a. M., 1970).

10 Dans le sens de Charles Grivel dans «Idée du texte», Romanistische Zeitschrift für Literaturgeschichte, Cahiers d'Histoire des Littératures Romanes (H.1-2, 1985) p. 162-179.

11 Je rappelle à ce propos différents types de lecteurs:

— «le lecteur explicite» (cf. Hans Robert Jauss, «Der Leser als Instanz einer neuen Geschichte der Literatur», Poetica 7 (H.3-4, 1975) p. 325-343).

- «le lecteur implicite» (cf. Wolfgang Iser, Der implizite Leser (München, 1972) p. 8 et suivantes).

— «le lecteur historique» (cf. Hans Ulrich Gumbrecht, «Konsequenzen der Rezeptionsästhetik oder Literaturwissenschaft als Kommunikationssoziolgie», Poetica 7 (H.3-4, 1975), p. 388-413, ici p. 390).

— «le lecteur intentionnel» (cf. E. Wolff, «Der intendierte Leser», Poetica 4 (1971) p. 141-166, ici p. 151).

- «le lecteur informé» (cf. Stanley Fish, «Literatur im Leser: Affektive Stilistik», dans Rainer Warning (éd.), Rezeptionsästhetik (München, 1975) p. 196-227).

— «'archi-lecteur» (cf. Michael Riffaterre, «Kriterien für die Stilanalyse», dans Rainer Warning (éd.), Rezeptionsästhetik (München, 1975) p. 163-195, ici p. 176).

Dans le contexte du modèle de notre pragmatique historique, nous concevons 
le «lecteur» comme «lecteur explicite» (H. R. Jauss) et comme «lecteur historique» (avec différentes dispositions de réception transmises socialement et historiquement).

\section{Charles Grivel, Le Fantastique (Mannheim, 1984) p. IV.}

13 En ce qui concerne la méthodologie de la pragmatique historique, $\mathrm{cf}$. Hans Ulrich Gumbrecht, "Receptie esthetica en handelingstheoretische literatuurwetenschap", dans Charles Grivel (éd.), Methoden in de literatuurwetenschap, op. cit. En ce qui concerne le statut de cette hypothèse de la fonction, cf. Hans Ulrich Gumbrecht et Jürgen E. Müller, «Sinnbildung als Sicherung der Lebenswelt», dans H.U. Gumbrecht, K. Stierle, R. Warning (éds.), Balzac - Ergebnisse eines romanistischen Kolloquiums (München, 1980) p. 339-389, en particulier p. 346 et p. 386.

Ence qui concerne cette fonction intentionnelle, il convient de rappeler ici qu'il nes'agit que très rarement d'une intention explicitement exprimée par l'auteur. Au contraire, en énonçant une telle hypothèse on se rapporte à une construction scientifique qui a pour objectif la reconstruction des motifs conscients et inconscients de la production et de la réception.

14 En ce qui concerne mon interprétation du terme «d'intermédialité», cf., par exemple, mon article «Texte et médialité», dans Jürgen E. Müller (éd.), Texte etmédialité, Mana VII (1987)p.9-13 ou mon article «Le labyrinthe médiatique du film: Helvyo Soto et "La triple mort du troisième personnage"», dans Michèle Biget, Nicole de Mourgues, Michel Servière (éds.), M.I.S. - Mots I Images / Sons (Rouen, 1990) p. 143-153.

\section{Dans le sens de la théorie phénoménologique.}

16 Des développements récents de la pragmatique historique tirent des conséquences de ce fait et incluent des réflexions de la théorie des systèmes dans leur cadre théorique et méthodologique. En ce qui concerne cette orientation, cf. Niklas Luhmann, Die Wissenschaft der Gesellschaft (Frankfurt a. M., 1990), en particulier p. 59 et suivantes. En ce qui concerne le statut de cette théorie dans le contexte d'une pragmatique historique, cf. M. Elsner, Th. Müller et P.M. Spangenberg, «Zur Entstehungsgeschichte des Dispositivs Fernsehen in der Bundesrepublik der fünfziger Jahre», in H. Kreuzer, H. Schanze (éds.), Ästhetik und Geschichte des bundesdeutschen Fernsehens, ici vol. I, K. Hickethier (éd.), Das Programm und seine Voraussetzungen (München, 1992).

Dans une version plus élaborée de cet article qui paraîtra dans Jürgen E. Müller (éd.), Towards a Pragmatics of the Audiovisual (Münster, 1992/93 (NODUS)), je vais ébaucher quelques liens entre la théorie pragmatique de Francesco Casetti, la théorie sémio pragmatique de Roger Odin et mon propos. Dans cette 
version, il y aura également deux schémas. Le premier concerne la communication textuelle et servira de démonstration didactique à mes explications sur la pragmatique historique du texte. Le second schéma servira à illustrer la communication filmique et audiovisuelle et à préciser quelques différences par rapport au schéma de la communication textuelle.

17 Sans partir explicitement de la théorie pragmatique, John Fiske, par exemple, expose dans son livre Television Culture (London/New York: Methuen, 1987) un point de vue intéressant sur l'interaction du contexte institutionnel et des schémas de réception des émissions de la télévision.

En ce qui concerne les dimensions psychiques et psychoanalytiques de ce processus, il convient de rappeler les analyses, entre-temps classiques, que Christian Metz expose dans son livre Le Signifiant imaginaire (Paris, 1977).

18 Pierre Melville, «La Nouvelle Vague, je l'ai inventée en 1937», Arts (25 avril 1962).

19 En ce qui concerne l'histoire du terme «Nouvelle Vague» et son fond historique dans la presse française (ce terme fut utilisé pour la première fois dans L'Express du 3 octobre 1957, visait la «nouvelle jeunesse des années 50", caractérisait ce que ressentait cette jeunesse, avant des'appliquer, explicitement, quelques mois plus tard, au cinéma de l'époque comme en témoignent L'Express et Cinéma), cf. Jean Pierre Nicolas, Le Cinéma des Français (Paris, 1979) p. 110. Nicolas nous renvoie dans son ouvrage à la «phraséologie de la nouveauté» comme unélément important du stock de savoir social de la société française vers la fin des années 50 .

20 Comparez la liste des régisseurs qui se trouve dans les Cahiers du cinéma, tome XXII, $n^{\circ} 138$ (décembre 1962). Dans ce tome se trouve une liste de «Cent soixante-deux nouveaux cinéastes français» (p. 60-94).

21 Dans le sens utilisé par Georg Schmid, «Geschichtsbilder. Von der Metaphorik zur Wörtlichkeit und retour», Zeitgeschichte 13(1986) p. 271-288.

22 Cf., par exemple, la liste des caractéristiques établie par Claire Clouzot dans son livre Le Cinéma français depuis la nouvelle vague (Paris, 1972) en particulier p. 23 et suivantes.

23 Cf., par exemple, David A. Cook, A History of Narrative Film (New York/ London, 1981) p. 462.

24 Ces éléments du stock social du savoir se distinguent biographiquement et socialement. En ce qui concerne cette question, cf. la théorie et la méthode de la sociologie de la connaissance élaborées par P. Berger et Th. Luckmann dans leur livre Diegesellschaftliche Konstruktionder Wirklichkeit (Frankfurt, 1970). 
En ce qui concerne la Nouvelle Vague, nous concevons (d'une manière provisoire et à cause des raisons «pragmatiques» de la recherche) ce groupe comme le cercle de la jeune génération autour de Bazin et comme le groupe des jeunes intellectuels et des cinéphiles autour de la Cinémathèque française et des ciné-clubs parisiens.

25 Georges Sadoul, Esprit, ${ }^{\circ} 6$ (juin 1960).

26 Michel Fabre, Présence du cinéma (mars-avril 1962).

27 François Truffaut, «Une certaine tendance du cinéma français», Cahiers du cinéma, $\mathrm{n}^{\circ} 31$ (1954) p. 15-28, ici p. 28.

28 J'emploie ici sciemment le terme «renaissance» de la notion d'auteur car cet aspect, extrêmement important pour l'histoire de la Nouvelle Vague, n'est pas vraiment aussi «nouveau» que le laisse croire le débat sur la Nouvelle Vague. En effet, Griffith, par exemple, ajoutait sa signature aux intertitres de ses films Intolerance et Birth of a Nation, ce qui devait en premier lieu éviter le plagiat et la piraterie. Cf. Cl. Gandelman et N. Greene, «Fétichisme, signature, cinéma», Hors Cadre 8, L'État d'auteur (1990) p. 147-161, ici p. 150. Cf. aussi l'article polémique de Pierre Melville, «La Nouvelle Vague, je l'ai inventée en 1937», Arts (25 avril 1962).

29 Roland Barthes, «La Mort de l'auteur», Manteia V (1968).

30 Dans le sens de la théorie phénoménologique de l'action d'Alfred Schütz et Thomas Luckmann. Schütz et Luckmann distinguent des «motifs du pour» («Um-Zu-Motive» - qui se dirigent sur un but de l'action) et des «motifs du parce que» («Weil-Motive» - qui se refèrent, sur la base des expériences et des attitudes qui se sont développées dans des situations, aux causes des actions). Cf. A. Schütz, Th. Luckmann, Strukturen derLebenswelt (Neuwied/Darmstadt, 1975) p. 213 et suivantes. En ce qui concerne l'intégration de ce concept dans le cadre d'une pragmatique historique, cf. H.U. Gumbrecht, «Receptie esthetica en handelingstheoretische literatuurwetenschap», op. cit.

31 Cf., par exemple, les textes rassemblés dans le volume de Jean Luc Douin (éd), La Nouvelle Vague 25 ans après (Paris, 1983).

32 J'emploie ce terme dans son sens phénoménologique, c'est-à-dire se rapportant à des motifs réels ou imaginaires de l'action.

33 Les implications de cette position dans la succession de la Nouvelle Vague sont démontrées par Tom Conley dans son article «L'auteur énucléé, Hors Cadre 8, L'État d'auteur (1990) p.77-95.

34 Dans le sens de Walter Benjamin, Das Kunstwerk im Zeitalter seiner 
technischen Reproduzierbarkeit (Frankfurt, 1977).

35 Dans le sens des «Relevanzstrukturen» de la thérorie d'Alfred Schütz et Thomas Luckmann, Strukturen der Lebenswelt, op. cit., p. 186 et suivantes.

36 Cf. Raymond Bellour, L'Entre-images (Paris: La Différence, 1990) p. 335 et suivantes; François Jost, «Der Picto-Film», dans J.E. Müller et M. Vorauer (éds.), Tendenzen im Spielfilm der 70-er und 80-er Jahre (Münster, 1992, NODUS) p. 223-236.

37 Cf. les remarques de Marc Bekaert dans son article «De Auteur als Theorie», Andere sinema, 98 (juli/augustus 1990) p. 40-45, surtout p. 42 et suivantes.

38 Notre petite étude de réception est basée sur un corpus de 17 critiques du film, qui sont parues dans des journaux néerlandais. Deux de ces articles (Parool, 26 mars 1960 et Volkskrant, 2 avril 1960) ont été rédigés par un correspondant néerlandais à Paris, cela ne fait aucun doute; en ce qui concerne un troisième (Haagse Post, 3 avril 1960), c'est très probable qu'il s'agisse dans ce cas-là, d'un article d'un correspondant à l'étranger. Je tiens à remercier les étudiants de mon séminaire sur la Nouvelle Vague, et tout particulièrement Laurence Lamers, du soutien qu'ils m'ont apporté pour rassembler les textes néerlandais.

39 Anthony Bosman, «Godard (26): eigen moraal. Àbout de souffle: hoogtepunt in film», Algemeen Dagblad (16 août 1960).

$40 \mathrm{Cf}$. les remarques que j'ai faites à ce sujet dans: Literaturwissenschaftliche Rezeptionstheorien und empirische Rezeptionsforschung - Mit einem Forschungsmodell, erläutert am Paradigma des französiischen Populärromans (Frankfurt a.M./Bern, 1981) p. 153 et suivantes.

41 À ce sujet, cf. aussi les remarques de Denise Pérusse dans son article «Réception critique et contexte:à propos du Déclindel'empireaméricain», dans le présent volume.

42 Cf., par exemple, H.U. Gumbrecht, «Literaturkritik als "Literatur"», W. Barner (éd.), Literaturkritik - Anspruch und Wirklichkeit (Stuttgart, 1991) p. 122-128.

43 «À bout de souffle», Vrije Volk (11 juin 1960).

44 On trouve, par exemple, juste sous le titre de l'article concacré à $\grave{A}$ bout de souffle l'information suivante imprimée en gras: «Jean-Luc Godard, Suisse, 26 ans, a pendant quelque temps écrit des textes pour des scénarios de films américains, a été peu de temps ouvrier sur un chantier naval, journaliste et en dernier lieu critique de cinéma». 
Anthony Bosman, «Godard (26): eigen moraal. À bout de souffle: hoogtepunt in film», Algemeen Dagblad (26 août 1960).

45 «Deux grands noms de la Nouvelle Vague — encore vague — de l'industrie cinématographique française sont liés au film $\grave{A}$ bout de souffle (Amour sans espérance) qui passe actuellement à l'ASTA: celui du François (Quatre cent coups) Truffaut qui a écrit le scénario et celui du Claude (Les Cousins) Chabrol qui en a dirigé la production. Ce n'est vraiment pas surprenant de voir que JeanLuc Godard (en tant que troisième ex-critique de cinéma) donne la main à des collègues plus célèbres que lui pour ta sortie de sa première œuvre: il n'est jamais facile de débuter.»

A. Pfeiffer, «À bout de souffle. Goede voorstelling van Jean-Luc Godard», Haags Dagblad (11 juin 1960).

46 «Een pretentieus debuut van Jean-Luc Godard», Nieuwe Rotterdamse Courant (27 août 1960).

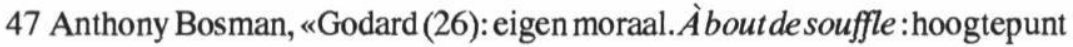
in film», Algemeen Dagblad (26 août 1960).

48 «À bout de souffle», Vrije Volk (11 juin 1960).

49 Cor de Groot, «Nieuwe golf zet door», Volkskrant (2 avril 1960).

50 J'utilise ce concept dans le sens de L'École de Prague.

51 «Het experiment in de speelfilm», NRC (11 juin 1960).

52 Il est ici fait allusion au fait que dans le paysage audiovisuel néerlandais de la fin des années 50 et du début des années 60 , les trois principaux piliers de la société néerlandaise («les catholiques», «les protestants-chrétiens» et les «socialistes») exerçaient encore une forte influence qui disparaîtra au cours des années suivantes. 